\title{
Symbolic dynamics and relatively hyperbolic groups
}

\author{
François Dahmani and Aslı Yaman
}

\begin{abstract}
We study the action of a relatively hyperbolic group on its boundary by methods of symbolic dynamics. We show that this dynamical system is expansive, and, under a condition on parabolic subgroups (satisfied in most examples), that it is finitely presented, meaning that it can be factorized through a subshift of finite type.
\end{abstract}

Mathematics Subject Classification (2000). 20F67, 37B10.

Keywords. Relatively hyperbolic groups, symbolic dynamics.

Let $\Gamma$ be a group and $\mathcal{A}$ a finite set (a set of symbols). The Bernoulli shift (or total shift) of $\Gamma$ on $\mathcal{A}$ consists of maps $\sigma: \Gamma \rightarrow \mathcal{A}$ and has the product topology. It is acted upon by $\Gamma$ as follows: $(\gamma \sigma)(g)=\sigma\left(\gamma^{-1} g\right)$ for all $g \in \Gamma$.

Consider a dynamical system: a group $\Gamma$ acting by homeomorphisms on a compact space $X$. A symbolic coding is an equivariant continuous surjection of a closed invariant subset (a subshift) of $\mathcal{A}^{\Gamma}$ to $X$. If the subshift in question is the smallest invariant containing the pre-image (for the projection map) of some set in $\mathcal{A}^{F}$, where $F$ is a finite subset of $\Gamma$, we say that the dynamical system is of finite type. If moreover it is expansive, it is said to be finitely presented (see references in the first section).

An application is that if a dynamical system is finitely presented, and if the preimage of each point of $X$ in the subshift is finite, certain counting functions, such as the Zeta function of the dynamical system, are rational [11].

The idea to define some $\Gamma$-equivariant coding of the elements of $X$ by elements of $\mathcal{A}^{\Gamma}$ has a long history. When $X$ is the boundary of a negatively curved space and $\Gamma$ is the discrete cocompact group of isometries of this space, a coding of the points of $X$ through the coding of the geodesic rays in the negatively curved space has been introduced and used by several authors, such as P. Koebe [13], G. Hedlund [12], M. Morse [14], [15], C. Series [16]. For the coarse version, i.e., for word-hyperbolic groups, these codings have been studied further by M. Gromov [11]. In this case $X$ is the boundary of a word-hyperbolic group. His argument given as an outline in [11] has been developed and presented in full details in the book of M. Coornaert and A. Papadopoulos; see [3], [4]. 
The proof presented by Coornaert and Papadopoulos strongly depends on local finiteness of the hyperbolic space and co-compactness of the action.

The aim of this paper is to state and prove similar codings for relatively hyperbolic groups, where parabolic subgroups are allowed. In general, in presence of parabolic elements, the study of dynamical properties becomes significantly more complicated.

The theory of relatively hyperbolic groups has been developed by B. Farb [9] and B. Bowditch [1] following the idea of Gromov in [11], as a generalization of geometrically finite Kleinian groups. We will use the definition given by Bowditch (See Definition 2.2). Bowditch's definition is equivalent to "relatively hyperbolic with the property BCP" of [9] (see the appendix of [5], and [7]).

Here are the most classical examples of relatively hyperbolic groups. Hyperbolic groups are hyperbolic relative to the empty set. To see that it suffices to replace "fine" by "locally finite" in Definition 2.2. In [9], Farb proves that the fundamental group of a finite volume manifold of pinched negative curvature, with finitely many cusps is hyperbolic relative to the conjugates of the fundamental groups of the cusps, which are virtually nilpotent. Sela's limit groups, or, finitely generated $\omega$-residually-free groups are hyperbolic relative to their maximal abelian non-cyclic subgroups, as shown in [6].

There is a well-defined notion of boundary for relatively hyperbolic groups, developed by B. Bowditch [1]. A relatively hyperbolic group acts on this compact space as a geometrically finite convergence group, and the elements of the family $\mathcal{E}$ are maximal parabolic subgroups for this action. We give here an intrinsic property of the maximal parabolic subgroups, namely the special symbol property defined as in Definition 1.5, that allows to pursue the method of Coornaert and Papadopoulos to find a finite coding of this action. A natural question related to this property is: which groups have the special symbol property? We prove in Section 4 that if a group has the special symbol property, then it is finitely generated. We also get a quite large family of examples.

Our main result is the following theorem.

Theorem 3.1. Let $(\Gamma, \mathcal{E})$ be a relatively hyperbolic group, and $\partial \Gamma$ be its boundary (in the sense of Bowditch [1]). If each $G \in \mathcal{E}$ has the special symbol property, then the action of $\Gamma$ on its boundary $\partial \Gamma$ is a finitely presented dynamical system.

One should remark that in [3] the subshift coding the action of a hyperbolic groups on its boundary enables algorithmic computations. Here, for simplicity, we give a non-constructive proof of the result, and we refer to the thesis of the second author ([18]) for a proof of the above theorem which enables computations. Another remark is that the coding we propose is not finite-to-one, leaving the question of the rationality of Zeta functions.

A group $\Gamma$ is said to be poly-hyperbolic if there is a sequence of subgroups $\{1\}=$ $N_{0} \triangleleft N_{1} \triangleleft \cdots \triangleleft N_{k-1} \triangleleft N_{k}=\Gamma$, with all the quotients $N_{i+1} / N_{i}$ hyperbolic. 
Theorem. Poly-hyperbolic groups have the special symbol property. In particular, this includes poly-cyclic groups.

Corollary. The action of a geometrically finite group of isometries of a simply connected manifold of pinched negative curvature (see [2]) on its limit set, is a finitely presented dynamical system.

We give definitions related to symbolic dynamics in Section 1. In Section 2, we define relatively hyperbolic groups, their boundaries and introduce some tools such as "angles" and "cones". We prove Theorem 3.1 in Section 3. The elements of our subshift are essentially local Busemann functions on the fine hyperbolic graph associated to the relatively hyperbolic group. To associate a point in the boundary to an element of the subshift, we consider its gradient lines. We prove that they converge to points at infinity, and we make sure that, for a given element of the subshift, all the gradient lines converge to the same point. For this we use the special symbol property for each stabilizer of infinite valence vertex.

We want to thank B. Bowditch, T. Delzant, and R. Grigorchuk for their comments and encouragements, M. Coornaert for his useful explanations, and the referee for his/her corrections and suggestions.

\section{Symbolic dynamics, definitions}

We borrow the following definitions (1.1 to 1.4) from Gromov [11] 8.4, and Coornaert and Papadopoulos [3], Chapter 2. See also Fried [10].

Definition 1.1 ([11], 8.4, [3], Chapter 2). If $\mathcal{A}$ is a finite discrete alphabet and $\Gamma$ is a group, $\mathcal{A}^{\Gamma}$, with the product topology, is the total shift of $\Gamma$ on $\mathcal{A}$. It admits a natural left $\Gamma$-action given by $(\gamma \sigma)(g)=\sigma\left(\gamma^{-1} g\right)$ for all $g \in \Gamma, \sigma \in \mathcal{A}^{\Gamma}$.

A closed $\Gamma$-invariant subset of $\mathcal{A}^{\Gamma}$ is called a subshift.

A cylinder $\mathcal{C}$ is a subset of the total shift such that there exists a finite set $F \subset \Gamma$, and a family of maps $M \subset \mathcal{A}^{F}$ with

$$
\mathcal{C}=\left\{\sigma \in \mathcal{A}^{\Gamma},\left.\sigma\right|_{F} \in M\right\} .
$$

A subshift $\Phi$ is a subshift of finite type if there exists a cylinder $\mathcal{C}$ such that $\Phi=\bigcap_{\gamma \in \Gamma} \gamma^{-1} \mathcal{C}$.

Definition 1.2. Let $\Gamma$ act on a compact set $K$. The dynamical system is of finite type if there exists a finite alphabet $\mathcal{A}$, a subshift of finite type $\Phi \subset \mathcal{A}^{\Gamma}$ and a continuous, surjective, $\Gamma$-equivariant map $\pi: \Phi \rightarrow K$. 
Example. Set $\Gamma=\mathbb{Z}$, and $\mathcal{A}=\{a, b, \$\}$. The cylinder $\mathcal{C}$ is the set of the maps that agree on $F=\{0,1\}$ with one of the maps in $M=\left\{m_{i}, i=1, \ldots, 4\right\}$ where $\left(m_{1}: 0,1 \rightarrow a, a\right),\left(m_{2}: 0,1 \rightarrow a, \$\right),\left(m_{3}: 0,1 \rightarrow \$, b\right)$ and $\left(m_{4}: 0,1 \rightarrow b, b\right)$.

Let $\Phi$ be the subshift of finite type defined by the cylinder $\mathcal{C}$, i.e., $\Phi=\bigcap_{n \in \mathbb{Z}} n+\mathcal{C}$, where $n+\mathcal{C}$ is the translate of the cylinder $\mathcal{C}$ of the total shift $\mathcal{A}^{\mathbb{Z}}$, by the element $n$ of $\mathbb{Z}$. One can check that the elements of $\Phi$ are the constant word on $a$, the constant word on $b$ and all the words (.. aaa $\$ b b b \ldots$ ) beginning by $a$, until there is a $\$$ on the $n$th letter $(n \in \mathbb{Z})$ and then $b$. Although for this example $\Phi$ is countable, in general subshifts of finite type are not countable.

Now consider the compact set $K=\mathbb{Z} \cup\{\infty\}$ with the usual topology. There is a natural left action of $\mathbb{Z}$ on $K$, fixing the infinity point. Consider the map $\pi: \Phi \rightarrow K$ that sends $(\ldots a a a a \ldots)$ and $(\ldots b b b b \ldots)$ to $\infty$, and $(\ldots a a a \$ b b b \ldots)$ to $n \in \mathbb{Z}$ where $n$ is the index of the letter $\$$. The map $\pi$ is surjective, continuous and equivariant, and therefore the action of $\mathbb{Z}$ on $K$ is of finite type.

We now continue with definitions. One can refine the property of being a dynamical system of finite type with the following.

Definition 1.3. The action of a group $\Gamma$ on a compact set $K$ is expansive if there exists $U$ a neighborhood of the diagonal $\Delta \subset K \times K$ such that $\Delta=\bigcap_{\gamma \in \Gamma} \gamma U$.

Note that, if the compact set is metric, this is equivalent to the property that there exists $\epsilon>0$ such that any two points can be sent at distance at least $\epsilon$ from each other by an element of the group (see [3], Proposition 2.3).

Definition 1.4. A dynamical system is finitely presented if it is both of finite type and expansive.

If one has a subshift of finite type $\Phi \subset \mathcal{A}^{\Gamma}$ and a surjective continuous equivariant map $\pi: \Phi \rightarrow K$, the expansivity of the action of $\Gamma$ on $K$ turns out to be equivalent to the fact that the subshift $\Psi \subset(\mathcal{A} \times \mathcal{A})^{\Gamma}$ defined by $\left[\left(\sigma_{1} \times \sigma_{2}\right) \in \Psi\right] \Leftrightarrow\left[\pi\left(\sigma_{1}\right)=\right.$ $\pi\left(\sigma_{2}\right)$ ], is of finite type (cf. [3], Chapter 2).

If $\Gamma$ is a infinite discrete group, it acts on its one-point compactification $\Gamma \cup\{\infty\}$ by multiplication on the left, hence fixing the point at infinity.

Definition 1.5. A discrete group $\Gamma$ has the special symbol property if its action by left multiplication on $\Gamma \cup\{\infty\}$ (or respectively on $\Gamma$ if $\Gamma$ is finite), is finitely presented by a subshift $\Phi \subset \mathcal{A}^{\Gamma}$ and if the coding map $\pi: \Phi \rightarrow \Gamma \cup\{\infty\}$ (resp. $\pi: \Phi \rightarrow \Gamma$ ) satisfies

$$
\exists \$ \in \mathcal{A} \quad(\pi(\sigma)=\gamma \in \Gamma) \Longleftrightarrow(\sigma(\gamma)=\$)
$$

In this case, since there are only two orbits of points, one of them consisting of isolated points, the property of expansivity of $\Gamma$ on $K=\Gamma \cup\{\infty\}$ is always satisfied. 
Thus, the left action of $\Gamma$ on $\Gamma \cup\{\infty\}$ is finitely presented if it is of finite type. The example of dynamical system of finite type of $\mathbb{Z}$ described above has the special symbol property. All finite groups also have it (more examples in Section 4).

For a group $\Gamma$ that has the special symbol property, let $\pi: \Phi \rightarrow \Gamma \cup\{\infty\}$ be a finite presentation with special symbol. Let $\mathcal{A}$ be the alphabet. Let $\mathcal{C}$ be a cylinder defining $\Phi$, and itself defined by a non-empty finite subset $F$ of $\Gamma$ and a set, $M$, of maps from $F$ to $\mathcal{A}$. Then we have the following result.

Proposition 1.6. If $\Gamma$ has the special symbol property, then $\Gamma$ is finitely generated and the finite set $F$ is a generating set for $\Gamma$.

Proof. The set of translates of $F$ is a covering of $\Gamma$. Let $P$ be the nerve of the covering. As $F$ is finite, $P$ is a finite dimensional, locally finite complex on which $\Gamma$ acts properly discontinuously cocompactly. The set of vertices of $P$ is naturally identified with $\Gamma$. The claim is that $P$ is connected. Otherwise it would have distinct connected components, $C_{i}$. Let $\gamma_{i}$ be a vertex of $C_{i}$, and consider $\sigma_{i} \in \Phi$ such that $\pi\left(\sigma_{i}\right)=\gamma_{i}$. Let $\sigma \in \mathcal{A}^{\Gamma}$ such that $\left.\left.\sigma\right|_{C_{i}} \equiv \sigma_{i}\right|_{C_{i}}$. Now, $\sigma$ has several special symbols (one in each $C_{i}$ ). On the other hand all the cylinder conditions defining $\Phi$ are satisfied, as by definition they are read on the connected components of $P$. This is a contradiction, and it proves the fact that $P$ is connected. Therefore, $\Gamma$ is generated by $F$ which is a finite set.

\section{About relatively hyperbolic groups}

2.1. Definitions. A graph is a set of vertices with a set of edges, where an edge is an unordered pair of vertices. We equip the geometrical realization of a graph with a metric where edges have length 1 .

In [1], B. Bowditch introduces the notion of fineness of a graph.

Definition 2.1 ([1]). A graph $\mathcal{K}$ is fine if for every $L>0$, and for every edge $e$, the set of simple simplicial loops of length less than $L$, containing $e$ is finite. It is uniformly fine if this set has cardinality bounded above by a constant depending only on $L$.

Definition 2.2 ([1]). A group $\Gamma$ is hyperbolic relative to a family of finitely generated subgroups $\mathcal{E}$, if it acts on a Gromov-hyperbolic, fine graph $\mathcal{K}$, with finite quotient, finite edge stabilizers, and such that the stabilizers of the vertices of infinite valence are exactly the elements of $\mathcal{E}$.

We also say, in this case, that the pair $(\Gamma, \mathcal{E})$ is a relatively hyperbolic group, and that $\mathcal{K}$ is a graph associated to it. 
We note that, since there are finitely many orbits of edges, a graph associated to a relatively hyperbolic group is uniformly fine. Let us also remark that the graph $\mathcal{K}$ associated to $(\Gamma, \mathcal{G})$ can be chosen to be without global cut point, and with positive hyperbolicity constant $\delta$ (as from the construction of the cone-off graph of B. Farb in [9]). Such a choice ensures that all the angles (introduced in the following section) are finite.

2.2. Angles. For any graph, one can define a notion of angle as follows.

Definition 2.3. Let $\mathcal{K}$ be a graph, and let $e_{1}=\left(v, v_{1}\right)$ and $e_{2}=\left(v, v_{2}\right)$ be edges. The angle $\operatorname{Ang}_{v}\left(e_{1}, e_{2}\right)$, is the length of the shortest path from $v_{1}$ to $v_{2}$, in $\mathcal{K} \backslash\{v\}$ $(+\infty$ if there is none).

The angle $\operatorname{Ang}_{v}\left(p, p^{\prime}\right)$ between two simple simplicial (oriented) paths $p$ and $p^{\prime}$, starting from a common vertex $v$, is the angle between their first edges after this vertex.

If $p$ is a simple simplicial path, and $v$ one of its vertices, not at the end of $p$, then $\operatorname{Ang}_{v}(p)$ is the angle between the consecutive edges of $p$ at $v$, and its maximal angle $\operatorname{MaxAng}(p)$ is the maximal angle between consecutive edges of $p$.

Proposition 2.4 (Some useful remarks).

$1 . \operatorname{Ang}_{v}\left(e_{1}, e_{3}\right) \leq \operatorname{Ang}_{v}\left(e_{1}, e_{2}\right)+\operatorname{Ang}_{v}\left(e_{2}, e_{3}\right)$ when $e_{i}$ are edges incident on a vertex $v$.

2. If $\gamma$ is an isometry of $\mathcal{K}, \operatorname{Ang}_{v}\left(e_{1}, e_{2}\right)=\operatorname{Ang}_{\gamma v}\left(\gamma e_{1}, \gamma e_{2}\right)$.

3. Any simple simplicial loop of length $L \geq 2$ has a maximal angle at most $L-2$.

Proof. The first remark is the triangular inequality for the length distance of $\mathcal{K} \backslash\{v\}$. The second statement is obvious. Finally, if $e_{1}=\left(v_{1}, v\right)$ and $e_{2}=\left(v, v_{2}\right)$ are two consecutive edges in a simple loop of length $L$, the loop itself gives a path of length $L-2$ from $v_{1}$ and $v_{2}$ avoiding $v$.

Lemma 2.5 (Large angles in triangles). Let $[x, y]$ and $[x, z]$ be geodesic segments in a $\delta$-hyperbolic graph, and assume that $\operatorname{Ang}_{x}([x, y],[x, z])=\theta \geq 50 \delta$. Then the concatenation of the two segments is still a geodesic. Moreover $x$ belongs to any geodesic segment $[y, z]$ and $\operatorname{Ang}_{x}([y, z]) \geq \theta-50 \delta$.

Proof. See Lemma 1.5 in [8].

\subsection{Cones}

Definition 2.6. Let $\mathcal{K}$ be a graph, let $d$ and $\theta$ be positive numbers. The cone centered at an edge $e=\left(v, v^{\prime}\right)$, of radius $d$ and angle $\theta$ is the set of vertices $w$ at distance at 
most $d$ from $v$ and such that there exists a geodesic segment $[v, w]$ the maximal angle and the angle with $e$ of which are at $\operatorname{most} \theta$, i.e.:

$$
\begin{aligned}
& \text { Cone }_{d, \theta}(e, v) \\
& \quad=\left\{w \in \mathcal{K} \mid \operatorname{dist}(w, v) \leq d, \exists[v, w], \operatorname{MaxAng}[v, w] \leq \theta, \operatorname{Ang}_{v}(e,[v, w]) \leq \theta\right\}
\end{aligned}
$$

Proposition 2.7 (Bounded angles imply local finiteness). Let $\mathcal{K}$ be a fine graph. Given an edge $e$ and $\theta>0$, there exists only finitely many edges $e^{\prime}$ such that $e$ and $e^{\prime}$ have a common vertex $v$, and $\operatorname{Ang}_{v}\left(e, e^{\prime}\right) \leq \theta$.

Proof. Only finitely many simple simplicial loops shorter than $(\theta+2)$ contain $e$.

Corollary 2.8 (Cones are finite). In a fine graph the cones are finite sets of vertices. If the graph is uniformly fine, the cardinality of $\operatorname{Cone}_{d, \theta}(e, v)$ can be bounded above by a function of $d$ and $\theta$.

Proof. Consider a cone Cone ${ }_{d, \theta}(e, v)$. We argue by induction on $d$. If $d=1$, the result is given by the previous proposition. If $d>1$, we remark that $\operatorname{Cone}_{d, \theta}(e, v)$ is contained in the union of cones of angle $\theta$ and radius 1 , centered at edges whose vertices are both in $\operatorname{Cone}_{(d-1), \theta}(e, v)$. If the latter is finite, the union is also finite.

Here is a visibility property for hyperbolic fine graphs. It is a usual result for proper hyperbolic graphs.

Proposition 2.9 (Visibility property in fine hyperbolic graphs). Let $\mathcal{K}$ be a hyperbolic fine graph and $\partial \mathcal{K}$ be its Gromov boundary. Then for all $\xi \in \partial \mathcal{K}$, and for all vertices $v$ in $\mathcal{K}$, there exists a geodesic ray $\rho=[v, \xi)$ in $\mathcal{K}$.

For all $\xi$ and $\xi^{\prime}$ in $\partial \mathcal{K}$, there exists a bi-infinite geodesic $\rho^{\prime}=\left(\xi, \xi^{\prime}\right)$ in $\mathcal{K}$.

Proof. Let $\left(v_{n}\right)$ be a sequence of vertices converging to $\xi$ in the sense of the Gromov topology. Consider two segments $\left[v, v_{n}\right]$, and $\left[v, v_{m}\right]$. If their angle at the vertex $v$ is greater than $50 \delta$, we can apply Lemma 2.5 to deduce that the Gromov product $\left(v_{n} \cdot v_{m}\right)_{v}$ is equal to zero. Therefore, there exists $n_{0}$ such that for all $n \geq n_{0}$, $A n g_{v}\left(\left[v, v_{n}\right],\left[v, v_{n_{0}}\right]\right) \leq 50 \delta$. Therefore, the first edges of these segments are all in a cone, and as the graph is fine, it is a finite set. After extraction of a subsequence, we can assume that these edges are all equal. The diagonal extraction process gives a subsequence $\left(v_{\sigma(n)}\right)_{n}$ such that $\left[v, v_{\sigma(n)}\right]$ coincide with $\left[v, v_{\sigma(m)}\right]$ for all $m \geq n$, on a subsegment $s_{n}$ of length $n$. As $s_{n}$ is a subsegment of $s_{m}$ for all $m \geq n$, their union is a geodesic ray $[v, \xi)$.

For the second assertion, we choose two rays $[v, \xi)$ and $\left[v, \xi^{\prime}\right)$. Let $\left(v_{n}\right)_{n}$ be a sequence of vertices that converges to $\xi$, on $[v, \xi)$ and $\left(v_{n}^{\prime}\right)_{n}$ another sequence that converges to $\xi^{\prime}$ on $\left[v, \xi^{\prime}\right)$. Let $d=\left(\xi \cdot \xi^{\prime}\right)_{v}+100 \delta$, and let $r$ and $r^{\prime}$ the subsegments of the rays $[v, \xi)$ and $\left[v, \xi^{\prime}\right)$, of length $d$. For all $n$ and $m$ sufficiently 
large, the geodesic segments $\left[v_{n}, v_{m}^{\prime}\right]$ intersect the cone of radius $d$ and of angle $\operatorname{Max} \operatorname{Ang}(r)+\operatorname{Max} \operatorname{Ang}\left(r^{\prime}\right)+A n g_{v}\left(r, r^{\prime}\right)+100 \delta$ centered at the first edge of $r$.

Therefore, as the cone is finite, one can find a subsequence of $v_{n}$ and $v_{n}^{\prime}$ and a point $p$ in the cone, such that every segment $\left[v_{n}, v_{m}^{\prime}\right]$ contains $p$. Now the diagonal process of the proof of the first assertion gives a sequence of segments converging to a bi-infinite geodesic.

As a corollary of the above proof we obtain the following result.

Corollary 2.10. Let $v$ be vertex in $\mathcal{K}$ and $\left(v_{n}\right)$ be a sequence of vertices converging to a point in $\partial \mathcal{K}$ in the sense of the Gromov topology. Then the edges of the geodesic segments $\left[v, v_{n}\right]$ adjacent to $v$ remain all in a cone for $n$ large enough. Moreover after extraction of a subsequence, we can assume that these edges are all equal.

We will use the following theorem, which is a reformulation of a result in [5].

Theorem 2.11. Let $\Gamma$ be a relatively hyperbolic group and $\mathcal{K}$ be an associated graph, which is $\delta$-hyperbolic. There exists an aspherical (in particular simply connected) simplicial complex such that its vertex set is the one of $\mathcal{K}$ and such that each simplex has all its vertices in a same cone of $\mathcal{K}$, of radius $10 \delta+10$ and angle $100 \delta+30$.

Proof. In [5], the first author defines the relative Rips complex $P_{d, r}(\mathcal{K})$ for a relatively hyperbolic group. It is the maximal complex on the set of vertices of $\mathcal{K}$ such that there is an edge between two vertices if, in $\mathcal{K}$, a geodesic of length at most $d$ and maximal angle at most $r$ links them. Although in [5], the notion of angle is replaced by "length of traveling in cosets", the proof of Theorem 6.2 remains the same, and gives the asphericity of $P_{d, r}(\mathcal{K})$ for radius $d \geq 10 \delta+10$ and angle $r \geq 100 \delta+30$. Theorem 2.11 follows.

2.4. Boundary of a relatively hyperbolic group. Let $(\Gamma, \mathcal{E})$ be a relatively hyperbolic group, and let $\mathcal{K}$ be an associated graph. In [1], Bowditch describes a model for the boundary $\partial \Gamma$ of $\Gamma$, as $\partial \Gamma=\partial \mathcal{K} \cup \mathcal{V}_{\infty}$ where $\partial \mathcal{K}$ is the Gromov boundary of the hyperbolic graph $\mathcal{K}$, and $\mathcal{V}_{\infty}$ is the set of vertices of infinite valence in $\mathcal{K}$. This boundary admits a natural topology of metrizable compact set (see [1], [17]). Let us recall a convergence criterion for this topology. Let $\left(v_{n}\right)_{n}$ be a sequence of vertices of infinite valence. If $\xi \in \partial \mathcal{K} \subset \partial \Gamma$, the sequence $v_{n}$ tend to $\xi$ if and only if the sequence of Gromov products $\left(v_{n} \cdot \xi\right)_{v_{0}}$ tends to infinity. If $v \in \partial \Gamma$ is a vertex of infinite valence, the sequence $v_{n}$ tends to $v$ if and only if almost every geodesic segments $\left[v_{0}, v_{n}\right]$ contains a vertex $v$, and $\operatorname{Ang}_{v}\left[v_{0}, v_{n}\right] \rightarrow \infty$. For a sequence $\left(\xi_{n}\right)_{n}$ of points in $\partial \mathcal{K}$, the conditions are similar: one needs only to change the segments $\left[v_{0}, v_{n}\right]$ into geodesic rays $\left[v_{0}, \xi_{n}\right]$.

The first result we have on the action of a relatively hyperbolic group on its boundary relates the expansivity property. 
Proposition 2.12 (Expansivity). The action of a relatively hyperbolic group on its boundary is expansive.

Proof. If $\Delta$ is the diagonal of $(\partial \Gamma) \times(\partial \Gamma)$, then we have to find a neighborhood $U$ of $\Delta$ such that $\Delta=\bigcap_{\gamma \in \Gamma} \gamma U$.

Let $\left\{e_{1}, \ldots, e_{m}\right\}$ be a set of orbit representatives of the edges in $\mathcal{K}$. Let $X$ be the set of pairs of points $\left(\xi_{1}, \xi_{2}\right) \in(\partial \mathcal{K})^{2}$ such that there is a bi-infinite geodesic between $\xi_{1}$ and $\xi_{2}$ passing through one of the $e_{i}$. Let now $\left\{p_{1}, \ldots, p_{m}\right\}$ be a set of orbit representatives of the infinite valence points. Because they are bounded parabolic points, the stabilizer $G_{i}$ of $p_{i}$ acts on $\partial \Gamma \backslash\left\{p_{i}\right\}$ with compact quotient. Let then $Y$ be the set of pairs of points $\left(p_{i}, \zeta\right)$ where $\zeta$ is in a chosen compact fundamental domain for the action of $G_{i}$.

Let $U=(\partial \Gamma \times \partial \Gamma) \backslash(X \cup Y)$. We claim that $\Delta=\bigcap_{\gamma \in \Gamma} \gamma U$.

Consider $\xi_{1} \neq \xi_{2}$. We consider two cases: either $\xi_{1}, \xi_{2}$ are both in $\partial \mathcal{K}$, or one them, say $\xi_{1}$, is a vertex of $\mathcal{K}$ of infinite valence. In the first case, there is a bi-infinite geodesic from one point to another, and it can be translated so that its image passes by one of the $e_{i}$. Therefore, there is $\gamma$ such that $\gamma\left(\xi_{1}, \xi_{2}\right)$ is in $X$, hence not in $U$. In the second case, there is $\gamma \in \Gamma$ such that $\gamma \xi_{1}$ is one of the $p_{i}$. Now there is $\gamma^{\prime} \in G_{i}$ such that $\gamma^{\prime} \gamma\left(\xi_{1}, \xi_{2}\right)$ is in $Y$, hence not in $U$. This proves that $\bigcap_{\gamma \in \Gamma} \gamma U \subset \Delta$, hence the claim.

Now we show that $U$ is a neighborhood of $\Delta$. That is to say that a sequence of elements in $X \cup Y$ cannot converge to a point of $\Delta$, in other words $X$ and $Y$ are closed.

Let $\left(x_{n}=\left(\xi_{1}^{n}, \xi_{2}^{n}\right)\right)_{n}$ be a converging sequence of elements of $X$. After passing to a subsequence, one can assume that, for all $n$, there is a bi-infinite geodesic between $\xi_{1}^{n}$ and $\xi_{2}^{n}$ passing through a same edge $e_{i}$. If $\xi_{1}^{n} \rightarrow \zeta_{1}$ and $\xi_{2}^{n} \rightarrow \zeta_{2}$, we see that $\zeta_{1}$ and $\zeta_{2}$ are linked by a geodesic passing through $e_{i}$, hence non-trivial. Therefore $\zeta_{1} \neq \zeta_{2}$.

Let now $\left(y_{n}=\left(\xi_{1}^{n}, \xi_{2}^{n}\right)\right)_{n}$ be a converging sequence of elements of $Y$. After extraction, and without loss of generality, one can assume that $\xi_{1}^{n}=p_{i}$, for all $n$, and for some $i$. Then, $\xi_{2}^{n}$ is in a compact fundamental domain for $G_{i}$ in $\partial \Gamma \backslash\left\{p_{i}\right\}$, and therefore does not converge to $p_{i}$. This finally proves that $U$ is a neighborhood of $\Delta$, and ends the proof of Proposition 2.12.

\section{Finite presentation of the boundary of a relatively hyperbolic group}

We will prove the following theorem.

Theorem 3.1. Let $(\Gamma, \mathcal{E})$ be a relatively hyperbolic group. If every group $G \in \mathcal{E}$ has the special symbol property, then the action of $\Gamma$ on its boundary $\partial \Gamma$ is finitely presented. 
3.1. Busemann and radial cocycles. Let $(\Gamma, \mathcal{G})$ be a relatively hyperbolic group, acting on some hyperbolic fine graph $\mathcal{K}$ with the properties of the definition. We call $\mathcal{V}$ the set of vertices of the graph $\mathcal{K}$.

Definition 3.2 ([11], 7.5.C, and [3], Chapter 3, Section 3). Let $\rho:[0, \infty) \rightarrow \mathcal{K}$ be a geodesic ray starting at $v_{0}$. The Busemann function $h_{\rho}: \mathcal{V} \rightarrow \mathbb{Z}$ of $\rho$ is defined by the limit (which always exists and is finite) $h_{\rho}(v)=\lim _{n \rightarrow \infty}(\operatorname{dist}(v, \rho(n))-n)$.

Definition 3.3 ([11], 7.5.E; [3], Chapter 3). Let $h_{\rho}$ be a Busemann function. The Busemann cocycle associated to $h_{\rho}$ is $\varphi_{\rho}: \mathcal{V} \times \mathcal{V} \rightarrow \mathbb{Z}$ defined by $\varphi_{\rho}(w, v)=$ $h_{\rho}(v)-h_{\rho}(w)$. A gradient line of $\varphi_{\rho}$ is a sequence of vertices $\left(v_{n}\right)_{n}$ such that $\varphi_{\rho}\left(v_{i+1}, v_{i}\right)=1$ for all $i$.

The first statement of the next lemma is Proposition 4.2 in [3], for locally finite graphs. In fact the proof does not refer to local finiteness and remains unchanged for our case. The proof of the second statement is done along the same lines as Proposition 3.4 in [3]. The proof of this proposition uses the visibility property of the boundary, which is satisfied here (Proposition 2.9).

Lemma 3.4. If $\varphi$ is a Busemann cocycle associated to $\rho$, then a gradient line is a sequence of vertices of a geodesic ray asymptotic to $\rho$. Moreover there is a gradient line starting from each vertex.

Proof. We only prove the second statement. Let $v$ be a vertex in $\mathcal{K}$. Consider the geodesic segments $[v, \rho(n)]$. Let $w_{n}$ be the vertex of $[v, \rho(n)]$ adjacent to $v$. Now by Corollary 2.10, after taking a subsequence $w_{n}=w$ for large enough $n$. Note that for these $n, \operatorname{dist}(v, \rho(n))=\operatorname{dist}(w, \rho(n))+1$. Thus $h_{\rho}(v)=h_{\rho}(w)+1$, and so $\varphi_{\rho}(w, v)=h_{\rho}(v)-h_{\rho}(w)=1$.

Definition 3.5. Let $p$ be a vertex of infinite valence in $\mathcal{K}$. The radial cocycle associated to $p$ is $\varphi_{p}: \mathcal{V} \times \mathcal{V} \rightarrow \mathbb{Z}$ defined by $\varphi_{p}(w, v)=\operatorname{dist}(v, p)-\operatorname{dist}(w, p)$. A gradient line of $\varphi_{p}$ is a finite family of vertices $\left(v_{n}\right)_{0 \leq n \leq m}$ such that $\varphi_{p}\left(v_{i+1}, v_{i}\right)=1$ for all $i$, and $v_{m}=p$.

The next lemma is direct by definition.

Lemma 3.6. If $\varphi$ is a radial cocycle associated to $p$, then its different gradient lines are exactly the sequences of vertices of geodesic segments ending at $p$.

Let $\delta$ be a positive hyperbolicity constant of $\mathcal{K}$. Without loss of generality, we can choose $\mathcal{K}$ without global cut point (for instance Farb's coned-off graph for a suitable system of generators), hence all angles are finite. Since there are only finitely orbits of 
finite valence vertices, there is a finite bound on the angle between two edges adjacent to a same vertex of finite valence. We set $\theta \geq 2000 \delta$ such a bound: any angle at any finite valence vertex is at most $\theta$.

Proposition 3.7 (Properties of Busemann and radial cocycles). Let $\varphi$ be a Busemann or a radial cocycle. Then:

(1) (Integral values) For $x, y$ adjacent vertices, $\varphi(x, y)$ is 0,1 or -1 (hence for all $x, y$ not necessarily adjacent, $|\varphi(x, y)| \leq \operatorname{dist}(x, y))$.

(2) (Cocycle) For all $x, y, z, \varphi(x, y)+\varphi(y, z)+\varphi(z, x)=0$.

(3) (Geodesic extension) Let $\xi$ be a point of $\partial \Gamma$, and let $l=[v, \xi)$ be a gradient line and $[x, v]$ a geodesic segment (of length and maximal angle at most $\theta$ ) such that $\operatorname{Ang}_{v}([x, v] \cup[v, \xi)) \geq \theta$. Then $[x, v] \cup[v, \xi)$ is a gradient line.

(4) (Exits) If $v$ is a vertex of finite valence, then there exists $w$ adjacent to $v$ with $\varphi(w, v)=1$.

Proof. Properties (1) and (2) are obvious. Property (4) is consequence of the Lemmas 3.4 and 3.6. Property (3) deserves a proof here. We emphasize that the assumption of maximal angle for the segment $[x, v]$ is unnecessary here, but will be useful elsewhere in this paper, where we prove that some other objects satisfy the same properties (see Lemma 3.12). By Lemma 3.4 (if $\xi \in \partial \mathcal{K}$ ) and Lemma 3.6 (if $\xi$ is a vertex of infinite valence), any gradient line from $x$ is a geodesic ray $[x, \xi)$ and produces a triangle $(x, v, \xi)$, which, by assumption, has a large angle at $v$. Hence, by Lemma 2.5, any ray $[x, \xi)$ contains $v$, in particular it is the case for any gradient line starting at $x$. This implies that $|\phi(v)-\phi(x)|=\operatorname{dist}(v, x)$, and therefore, the segment $[x, v]$ belongs to a gradient line. This implies that $[x, v] \cup[v, \xi)$ is a gradient line.

3.2. Shift and subshift. We keep the notations of the previous section: $\Gamma$ is a relatively hyperbolic group, and $\mathcal{K}$ is a hyperbolic fine graph, acted upon by $\Gamma$ as in the definition of relative hyperbolicity. Since $\Gamma$ acts on the graph $\mathcal{K}$ with finite quotient, the number of conjugacy classes of maximal parabolic subgroups is finite. Let $\left(G_{i}\right)_{i=1, \ldots, m}$ be a finite family of representatives of these conjugacy classes in $\Gamma$. For each $i$, the group $G_{i}$ fixes an infinite valence vertex $p_{i}$ in $\mathcal{K}$. We also choose, for each $i \leq m$, an arbitrary edge $e_{i}$ in $\mathcal{K}$, adjacent to $p_{i}$.

We make the assumption (as in Theorem 3.1) that each group $G_{i}$ has the special symbol property. Let $\Phi_{i} \subset \mathcal{A}_{i}^{G_{i}}$ be a subshift of finite type as in Definition 1.5 , let $F_{i}$ be a finite subset of $G_{i}$ and $M_{i}$ a set of maps from $F_{i}$ to $\mathcal{A}_{i}$ defining the cylinder $\bigodot_{i}$ such that $\Phi_{i}=\bigcap_{\gamma \in G_{i}} \gamma^{-1} \bigodot_{i}$. In what follows we shall omit the index $i$ when mentioning the special symbol $\$$ in $\mathcal{A}_{i}$.

Without loss of generality, we can assume that the graph $\mathcal{K}$ is such that for all $i$, and for all $\gamma \in F_{i}, \operatorname{Ang}_{p_{i}}\left(e_{i}, \gamma e_{i}\right) \leq 1$, and, as before, without cut point (for example, 
one can take Farb's coned-off graph for a suitable generating set, see [9]). We keep $\theta \geq 2000 \delta$ as in the previous section.

For all $i=1, \ldots, m$ we set $F_{i}^{\prime}=\left\{\gamma \in G_{i} \mid \operatorname{Ang}_{p_{i}}\left(e_{i}, \gamma e_{i}\right) \leq \theta / 2\right\}$. This set contains $F_{i}$.

We fix a vertex $v_{0}$ and an edge $e_{0}=\left(v_{0}, v\right)$. We choose $R$ and $\Theta$ sufficiently large, such that for all $i=1, \ldots, m$, Cone ${ }_{10 \theta, 10 \theta}\left(e_{i}, p_{i}\right) \subset$ Cone $_{R, \Theta}\left(e_{0}, v_{0}\right)$. Note that by the choice of $\theta, R$ and $\Theta$ are greater than $(100 \delta+30)$, the constant given by Theorem 2.11.

Let $\mathcal{A}^{\prime}$ denote the set of all possible restrictions of Busemann and radial cocycles on Cone ${ }_{R, \Theta}\left(e_{0}, v_{0}\right) \times$ Cone $_{R, \Theta}\left(e_{0}, v_{0}\right)$. We set $\mathcal{A}^{\prime \prime}=\mathcal{A}_{1} \times \cdots \times \mathcal{A}_{m}$. We choose our alphabet to be $\mathcal{A}=\mathcal{A}^{\prime} \times \mathcal{A}^{\prime \prime}$

Lemma 3.8. $\mathcal{A}=\mathcal{A}^{\prime} \times \mathcal{A}^{\prime \prime}$ is finite.

Proof. Cones are finite, and cocycles have integral values bounded by the diameter of the cones.

Any element $\psi$ of $\mathcal{A}^{\Gamma}$ is a map from $\Gamma$ to $\mathcal{A}=\mathcal{A}^{\prime} \times \mathcal{A}^{\prime \prime}$; thus it has coordinates $\psi_{0}: \Gamma \rightarrow \mathcal{A}^{\prime}$ and $\psi_{i}: \Gamma \rightarrow \mathcal{A}_{i}$ for all $i$. Hence, $\psi_{0}(\gamma)$ is a map from Cone $_{R, \Theta}\left(e_{0}, v_{0}\right) \times$ Cone $_{R, \Theta}\left(e_{0}, v_{0}\right)$ to $\mathbb{Z}$, whereas $\psi_{i}(\gamma)$ is in $\mathcal{A}_{i}$ for $i \geq 1$.

Let $F$ be the set of elements in $\Gamma$ such that the endpoints of $\gamma e_{0}$ are both in Cone $_{R, \Theta}\left(e_{0}, v_{0}\right)$. As stabilizers of edges are finite, $F$ is a finite set.

Let $\mathcal{C}$ be the cylinder (in the sense of Definition 1.1) defined on $F$ so that $\psi \in \mathcal{C}$ if the following three conditions, which concern only finitely many elements of $\Gamma$, are fulfilled:

(a) $\left[\psi_{0}(\gamma)\right]\left(v_{1}, v_{2}\right)=\left[\psi_{0}\left(1_{\Gamma}\right)\right]\left(\gamma^{-1} v_{1}, \gamma^{-1} v_{2}\right)$ whenever $v_{1}, v_{2}, \gamma^{-1} v_{1}, \gamma^{-1} v_{2}$ are all in Cone C,$\Theta\left(e_{0}, v_{0}\right)$.

(b) $\left.\psi_{i}\right|_{F_{i}}$ is in $M_{i}$.

(c) for $\gamma \in F_{i}$, for $v$ such that $\gamma e_{i}=\left(p_{i}, v\right)$, and for $w$ such that $\left[w, p_{i}\right]$ is a geodesic segment of length, and maximal angle, at most $\theta$, containing $v$, one has $\left[\psi_{0}(\gamma)\right]\left(\gamma^{-1} w, p_{i}\right) \geq\left(1-\operatorname{dist}\left(w, p_{i}\right)\right)$ only if there exists $\gamma^{\prime} \in F_{i}^{\prime}$ such that $\psi_{i}\left(\gamma \gamma^{\prime}\right)=\$$.

Denote by $\Phi$ the subshift of finite type $\Phi=\bigcap_{\gamma \in \Gamma} \gamma \mathcal{C}$.

We will see in the next lemmas that the property (b) of the subshift allows us to say that, at a vertex of infinite valence, there is at most one edge $e$ issued from this vertex, such that the $i$ th coordinate of $\psi$ takes the symbol $\$$ on the other vertex of $e$. Moreover the property (c) of the subshift will ensure that the gradient lines escape a vertex of infinite valence only around the edge which takes the symbol \$.

Lemma 3.9 (Globalization). Let $\psi \in \Phi$. If $v$ and $v^{\prime}$ are vertices in $\gamma$ Cone $_{R, \Theta}\left(e_{0}, v_{0}\right)$, we set $\varphi_{\psi}\left(v, v^{\prime}\right)=\psi_{0}(\gamma)\left(\gamma^{-1} v, \gamma^{-1} v^{\prime}\right)$. Then the map $\varphi_{\psi}$ is well defined. Moreover 
$\varphi_{\psi}$ can be extended to all pairs of vertices of $\mathcal{K}$, and is a global cocycle that takes values in $\mathbb{Z}$.

Proof. Because of the property (a) of the definition of $\ell$, the formula given for $\varphi_{\psi}\left(v, v^{\prime}\right)$ does not depend on the choice of possible $\gamma$, and therefore, the map is well defined. The map $\varphi_{\psi}$ is defined on pairs of vertices lying in a same translate of Cone $_{R, \Theta}\left(e_{0}, v_{0}\right)$. By definition of the alphabet, $\varphi_{\psi}$ is, on each of these translates, the restriction of a certain Busemann or a radial cocycle (in particular it has the cocycle property on each of these finite sets). Thus $\varphi_{\psi}$ is a 1-cochain defined on the relative Rips complex $P$ given by Theorem 2.11. Since the simplices of $P$ are vertices in a same translate of Cone $\operatorname{Co}_{, \Theta}\left(e_{0}, v_{0}\right)$, the local cocycle property mentioned above ensures that $\varphi_{\psi}$ is a cocycle on $P$. By Theorem $2.11, P$ is simply connected, hence $\varphi_{\psi}$ is a co-boundary: there is a map $\tilde{\varphi}$ defined on the set of vertices of $\mathcal{K}$ such that $\varphi_{\psi}(w, v)=\tilde{\varphi}(w)-\tilde{\varphi}(v)$ for all $v, w$ lying in a translate of Cone $\operatorname{Co}_{\Theta}\left(e_{0}, v_{0}\right)$. This formula allows to extend the cocycle $\varphi_{\psi}$ to all pairs of vertices. By the first property of Proposition 3.7, it takes values in $\mathbb{Z}$.

From now on we will assume that $\varphi_{\psi}$ is defined globally.

Lemma 3.10 (About the $\psi_{i}, i \geq 1$ ). Let $\psi \in \Phi$ and $\gamma \in \Gamma$. Then for all $i$, the map defined on $G_{i}: g_{i} \mapsto \psi_{i}\left(\gamma g_{i}\right)$ is an element of $\Phi_{i}$.

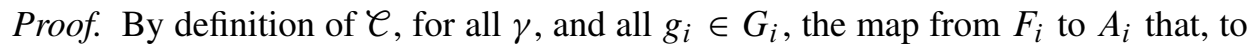
$f$ associates $\psi_{i}\left(\gamma g_{i} f\right)$, is in $M_{i}$.

For the moment, we have gradient lines only for Busemann and radial cocycles.

Definition 3.11. Let $\psi$ be an element of $\Phi$. A gradient line $l_{\psi}$ of $\psi$ is a maximal (finite or infinite) sequence $\left(v_{n}\right)_{n \geq 0}$ of adjacent vertices in $\mathcal{K}$ such that $\varphi_{\psi}\left(v_{n+1}, v_{n}\right)=1$ for all $n$.

Lemma 3.12 (About $\psi_{0}$ ). For all $\psi \in \Phi, \varphi_{\psi}$ satisfies each property of Proposition 3.7 and $\left|\varphi_{\psi}(w, v)\right| \leq \operatorname{dist}(w, v)$ for all $v, w$.

Proof. Let $v=v_{0}, v_{1}, \ldots, v_{L}=w$ be consecutive vertices in a geodesic segment. We have $\left|\varphi_{\psi}\left(v_{i+1}, v_{i}\right)\right| \leq 1$ for all $i$. Since $\varphi_{\psi}$ is a global cocycle, $\left|\varphi_{\psi}\left(v_{0}, v_{L}\right)\right| \leq L$, hence the last claim. Properties 1 and 4 of Proposition 3.7 are satisfied because each element of our alphabet satisfies them in a cone. Property 2 (cocycle property) was proved in Lemma 3.9. Let us prove the third property. Let us notice first that, by Definition 1.5, for all $\gamma_{0} \in \Gamma$, the symbol $\$$ can appear at most once in the set of values $\left\{\psi_{i}\left(\gamma_{0} \gamma\right), \gamma \in G_{i}\right\}$. If $[v, \xi)$ is a gradient line, with $v=\gamma_{0} p_{i}$, for some $i$ and some $\gamma_{0}$, then for $\gamma$ such that $\psi_{i}\left(\gamma_{0} \gamma\right)=\$$, there exists $\gamma^{\prime}$ in $F_{i}^{\prime}$ such that $\left(\gamma_{0} \gamma \gamma^{\prime}\right) e_{i}$ is the first edge of $[v, \xi)$. If $[v, x]$ is a segment such that $\operatorname{Ang}_{v}([v, x],[v, \xi)) \geq \theta$, 
then, for all $\gamma^{\prime \prime} \in F_{i}^{\prime}$, the edge $\left(\gamma_{0} \gamma \gamma^{\prime \prime}\right) e_{i}$ is not on $[v, x]$. Therefore, by the property (c) of the definition of the cylinder, $\varphi_{\psi}(x, v) \leq-\operatorname{dist}\left(x, p_{i}\right)$. And this, together with $\left|\varphi_{\psi}(x, v)\right| \leq \operatorname{dist}(x, v)$, gives $\varphi_{\psi}(x, v)=-\operatorname{dist}\left(x, p_{i}\right)$. In other words, $[x, v] \cup[v, \xi)$ is a gradient line.

3.3. The presentation $\Pi: \Phi \rightarrow \partial \Gamma$. Given an element of $\Phi$, we want to associate an element of $\partial \Gamma$ in a $\Gamma$-equivariant way.

Lemma 3.13. The gradient lines of the elements of $\Phi$ are geodesics in $\mathcal{K}$.

Proof. Let $\psi$ be an element of $\Phi$. It suffices to prove that $\left|\varphi_{\psi}(v, w)\right|=\operatorname{dist}(v, w)$, for all $v$ and $w$ on a gradient line $l_{\psi}$. With the notation of the definition, let $v=v_{n}$ and $w=v_{m}$ be two such points. Thus, we have $\varphi_{\psi}\left(v_{n+k+1}, v_{n+k}\right)=1$ for every $k$. Since $\varphi_{\psi}$ is a global cocycle, $\varphi_{\psi}\left(v_{m}, v_{n}\right)=m-n$. Moreover, the gradient line $l_{\psi}$ provides a path of length $n-m$ between $v_{n}$ and $v_{m}$, therefore, $\operatorname{dist}\left(v_{n}, v_{m}\right) \leq$ $|m-n|=\left|\varphi_{\psi}\left(v_{m}, v_{n}\right)\right|$. By Lemma 3.12, we get the other inequality.

We now state and prove the main property of the elements of $\Phi$. In order to unify the terminology for infinite and finite gradient lines, we say that a finite gradient line converges to a point $v \in \mathcal{V}_{\infty}$ if $v$ is its endpoint.

Proposition 3.14 (Coherence of gradient lines). Let $\psi \in \Phi$. All its gradient lines are asymptotic to each other. In other words they all converge to the same element of $\partial \mathcal{K} \cup \mathcal{V}_{\infty}$.

Before giving the proof of Proposition 3.14 we need some preliminary lemmas. We say that two gradient lines of $\psi$ are divergent if they have different endpoints in the boundary of $\mathcal{K}$ or in the set of vertices of infinite valence $\mathcal{V}_{\infty}$.

Lemma 3.15. Given two divergent gradient lines of $\psi$ one can find two divergent gradient lines of $\psi$ starting at the same vertex, or at two adjacent vertices.

Proof. Let $l_{1}$ and $l_{2}$ be two divergent gradient lines, and $v_{1}$ and $v_{2}$ vertices on them. On a geodesic segment $\left[v_{1}, v_{2}\right]$, consider $v$ the first vertex from which there is a gradient line $l$ divergent from $l_{1}$. Either $v=v_{1}$ (and we are in the first case of the lemma), or there is a vertex, $v^{\prime}$, of $\left[v_{1}, v\right]$ adjacent to $v$. By definition of $v$, all gradient lines starting at $v^{\prime}$ are asymptotic to $l_{1}$, and we are in the second case of the lemma.

Let $l_{1}, l_{2}$ and $l_{3}$ be three geodesic lines connecting respectively $x_{3}$ with $x_{1}, x_{3}$ with $x_{2}$ and $x_{1}$ with $x_{2}$, and suppose $l_{1}$ and $l_{2}$ are divergent gradient lines (possibly infinite) for $\psi$. 
Lemma 3.16. Let $v$ be a vertex of $l_{1}$ such that $\left(x_{1} \cdot x_{2}\right)_{x_{3}}-50 \delta<\operatorname{dist}\left(x_{3}, v\right)<$ $\left(x_{1} \cdot x_{2}\right)_{x_{3}}+50 \delta$. If $v$ is not on $l_{3}$, and if $\operatorname{Ang}_{v}\left(l_{1}\right)$ is more than $5 \theta$ then the line $l_{2}$ passes through $v$ and $\operatorname{Ang}_{v}\left(\left.l_{1}\right|_{\left[v, x_{1}\right]},\left.l_{2}\right|_{\left[v, x_{2}\right]}\right) \leq \theta$, where the paths involved are the sub-paths of $l_{1}$ and $l_{2}$ that are after $v$. In this case, $d\left(x_{3}, v\right) \geq\left(x_{1} \cdot x_{2}\right)_{x_{3}}$.

Proof. The second assertion is an easy consequence of the first one. It is enough to show that $v$ is on $l_{2}$, as we know by Lemma 3.12 that two gradient lines starting at the same point make an angle at this point smaller than $\theta$.

At distance $\left(x_{1} \cdot x_{2}\right)_{x_{3}}-100 \delta$ from $x_{3}$, we connect $l_{1}$ and $l_{2}$ by a segment $\alpha_{3}$ of length at most $10 \delta$, and we connect $l_{i}$ to $l_{3},(i=1,2)$ at distance $\min \left\{\left(x_{1} \cdot x_{2}\right)_{x_{3}}+\right.$ $\left.100 \delta, d\left(x_{3}, x_{i}\right)\right\}$ from $x_{3}$ by another segment $\alpha_{i}$, of length at most $10 \delta$.

Following subsegments of the triangle, and the segments $\alpha_{i}$, we get a loop of length at most $2 \times(3 \times 10 \delta+2 \times 100 \delta)$, that passes by $v$ once. The result follows from an angle chase in this loop.

Proof of Proposition 3.14. We argue by contradiction, and assume that $l_{1}$ and $l_{2}$ are two divergent gradient lines. By Lemma 3.15 we can assume that $l_{1}$ and $l_{2}$ are two divergent gradient lines starting at the same vertex, or at two adjacent vertices. In order to simplify the presentation we will only explicitly treat the first case as the second case can be treated exactly with the same arguments. Thus by Proposition 2.9, there is a geodesic (possibly bi-infinite) $l_{3}$, such that $\left(l_{1}, l_{2}, l_{3}\right)$ is a geodesic triangle with vertices $x_{1}, x_{2}, x_{3}$ (see Figure 1), with $x_{1}$ and $x_{2}$ possibly at infinity.

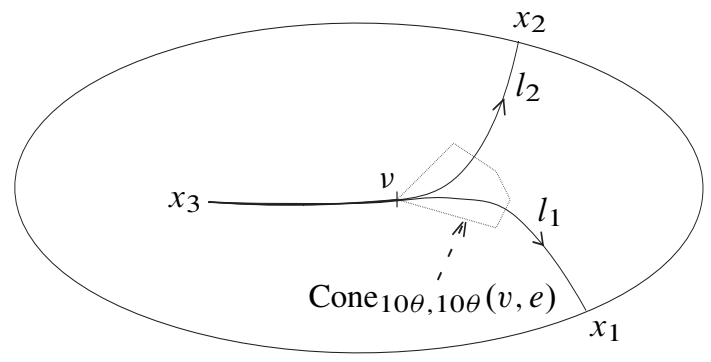

Figure 1. Gradient lines and cone at the center of the triangle.

Let $v$ be the vertex satisfying the assumptions of the previous lemma, that maximizes $d\left(x_{3}, v\right)$ (or, if there is none, the vertex on $l_{1}$ at distance $\left(x_{1} \cdot x_{2}\right)_{x_{3}}-50 \delta$ from $\left.x_{3}\right)$. By the previous lemma, and by maximality of the distance $d\left(x_{3}, v\right)$, the two rays $l_{1}$ and $l_{2}$ do not have an angle larger than $5 \theta$ after $v$, until they arrive at distance $50 \delta$ from the small segments connecting $l_{1}, l_{3}$, and $l_{2}, l_{3}$.

Consider the cone $C$ centered on the first edge of $l_{1}$ after our vertex $v$, of angle and radius $10 \theta$. Let us parameterize by arc length the two subsegments intersecting this cone $C$ of the two lines $l_{1}$ and $l_{2}$. Let $\lambda_{i}:\left[0, T_{i}\right] \rightarrow \operatorname{Cone}_{10 \theta, 10 \theta}(v, e)$, for $i=1,2$ 
be such parameterizations where $T_{i}$ is the length of the segment of $l_{i} \cap C$. Note that $\lambda_{i}(0)=v$. We know that $\operatorname{dist}\left(\lambda_{1}(0), \lambda_{2}(0)\right) \leq \delta$, because the triangle $\left(l_{1}, l_{2}, l_{3}\right)$ is $\delta$ thin. Moreover, as $\operatorname{Ang}_{v}\left(\lambda_{1}, \lambda_{2}\right) \leq \theta$ (by 3.16) and as the two rays do not have angle larger than $5 \theta$ after $v$, until they arrive close to the transition segments, the vertices $\lambda_{1}\left(T_{1}\right)$ and $\lambda_{2}\left(T_{2}\right)$ are at distance at least $\left(x_{1} \cdot x_{2}\right)_{x_{3}}+20 \delta$ from $x_{3}$, and therefore, at distance at least $35 \delta$ from each other (see Figure 1 ) - or possibly a segment $\lambda_{i}$ reaches $x_{i}$, which, in this case, belongs to the cone.

By definition of our alphabet $\mathcal{A}$, there must be a Busemann or a radial cocycle whose restriction on this cone induces the same segments of gradient lines. This rules out the second case, and in the first case, by hyperbolicity, two geodesic rays with such subsegments would diverge at infinity, but this contradicts the uniqueness of the end points of the gradient lines of Busemann and radial cocycle.

We can now define the map $\Pi: \Phi \rightarrow \partial \Gamma$. To an element $\psi$ in $\Phi$, we associate the point $\Pi(\psi) \in \partial \Gamma$ to which any gradient line of $\varphi_{\psi}$ converges.

3.4. End of the proof of Theorem 3.1. In order to complete the proof we need to show that $\Pi: \Phi \rightarrow \partial \Gamma$ satisfies Definition 1.2 (Lemma 3.17), and secondly that the action of $\Gamma$ on $\partial \mathcal{K}$ is expansive (Proposition 2.12).

Lemma 3.17. The map $\Pi: \Phi \rightarrow \partial \Gamma$ is surjective, continuous and equivariant.

Proof. Given a point $\xi$ in $\partial \Gamma$, by the visibility property, one can find a Busemann or a radial cocycle $\sigma$ associated to $\xi$. We consider the function $\tilde{\sigma}: \Gamma \rightarrow \mathcal{A}^{\prime}$ obtained by $\tilde{\sigma}(\gamma)=\left.\sigma\right|_{\left(\gamma \text { Cone }_{R, \Theta}\left(e_{0}, v_{0}\right)\right)^{2}}$. Moreover, for every $i$, and for every left $\operatorname{coset} \gamma G_{i}$ $(i=1, \ldots, m)$, we fix a representative $\gamma g_{i}$. For every $i$, and every $\gamma g_{i}$ in the set of chosen representatives, we consider the function $\psi_{i, \gamma g_{i}}: G_{i} \rightarrow \mathcal{A}_{i}$, in $\Phi_{i}$ such that it takes the value of the special symbol only for an element $g \in G_{i}$ such that $\tilde{\sigma}\left(\left(\gamma g_{i} g\right)^{-1} v, p_{i}\right)<0$, for some vertex $v$ adjacent to $\left(\gamma g_{i} g\right) p_{i}$.

For each $i$, the partition of $\Gamma$ in $G_{i}$-left cosets defines a function $\tilde{\psi}_{i}: \Gamma \rightarrow \mathcal{A}_{i}$, that associates, to $\gamma=\gamma g_{i} g, g \in G_{i}$, the value $\psi_{i, \gamma g_{i}}(g)$.

This gives a function $\varphi: \Gamma \rightarrow \mathcal{A}$, namely the product of $\tilde{\sigma}$ and of the $\tilde{\psi}_{i}, i=$ $1, \ldots, m$ which is, by Proposition 3.7, an element of $\Phi$ whose gradient lines converge to $\xi$. Thus, the map $\Pi$ is surjective.

If a sequence $\psi_{n}$ of elements of $\Phi$ converges to $\psi$, then, given a finite set $E$ of edges of $\mathcal{K}$, the gradient lines of $\psi_{n}$ will coincide with the gradient lines of $\psi$ on $E$ for $n$ sufficiently large. If the point at infinity $\xi$ defined by $\psi$ is in $\partial \mathcal{K}$, then by the usual topology on the boundary of $\mathcal{K}$, the sequence of points at infinity $\xi_{n}$, defined by the gradient lines of $\psi_{n}$, converges to $\xi$. If now $\xi$ is in $\mathcal{V}_{\infty}$, then, by convergence criterion given in paragraph 2.4, the sequence $\xi_{n}$ converges to $\xi$. This ensures the continuity of $\Pi$. 
Finally, if $\psi$ is an element of $\Phi$, and $\rho$ one of its gradient lines, then $\gamma \rho$ is a gradient line for $\gamma \psi$. The point at infinity of $\gamma \rho$ is by definition $\gamma \Pi(\psi)$, and it is therefore equal to $\Pi(\gamma \psi)$. Hence the map is equivariant.

\section{Groups having the special symbol property}

We now give examples of groups with the special symbol property. Let us begin with a necessary condition.

The next proposition is in fact is a slight variation of a theorem of Gromov, a detailed proof of which can be found in [3] (Corollary 8.2).

Proposition 4.1. If $\Gamma$ is a hyperbolic group, then it has the special symbol property.

Proof. We repeat the proof of the main theorem, seeing $\Gamma$ hyperbolic relative to the trivial subgroup $\{1\}$. A Cayley graph plays the role of $\mathcal{K}$, and we consider the same cocycles. They can define either a point at infinity, or a vertex of the graph. Thus, we obtain our presentation choosing the special symbol to be the restriction of a radial cocycle.

The example in part 1 already provided the basic examples of $\mathbb{Z}$ and of finite groups, as groups with the special symbol property. Most of our remaining examples come from the following remarks.

Proposition 4.2. If a group $\Gamma$ splits in a short exact sequence $\{1\} \rightarrow N \rightarrow \Gamma \rightarrow$ $H \rightarrow\{1\}$, and if both $N$ and $H$ have the special symbol property, then $\Gamma$ has the special symbol property.

Proposition 4.3. Let $G$ be a subgroup of finite index of a group $\Gamma$. The group $G$ has the special symbol property if and only if $\Gamma$ has the special symbol property.

Before giving the proofs, we give a consequence. A group $\Gamma$ is said to be polyhyperbolic if there is a sequence of subgroups $\{1\}=N_{0} \triangleleft N_{1} \triangleleft \cdots \triangleleft N_{k-1} \triangleleft$ $N_{k}=\Gamma$, with all the quotients $N_{i+1} / N_{i}$ hyperbolic.

Corollary 4.4. Every poly-hyperbolic group has the special symbol property. In particular, this includes virtually polycyclic (hence, all finitely generated, virtually nilpotent) groups.

Proof. If $\Gamma$ is poly-hyperbolic, there is a sequence of subgroups $\{1\}=N_{0} \triangleleft N_{1} \triangleleft$ $\cdots \triangleleft N_{k-1} \triangleleft N_{k}=\Gamma$, with all the quotients $N_{i+1} / N_{i}$ hyperbolic. Using the Proposition 4.2, and the fact that hyperbolic groups have the special symbol property, an induction on $i$ tells that each $N_{i}$ has the special symbol property, and especially $N_{k}$ which is $\Gamma$. 
Proof of Proposition 4.2. Let us denote by $\mathcal{A}_{N}, \mathcal{A}_{H}, \$_{N}, \$_{H}, \mathcal{C}_{N}, \mathcal{C}_{H}, \Phi_{N}, \Phi_{H}$ the alphabets, special symbols, cylinders, and subshifts of finite type for the presentations of $N \cup\{\infty\}$ and $H \cup\{\infty\}$. Let $F_{N}, F_{H}, M_{N}$ and $M_{H}$ be the finite subsets of $N$ and $H$, and respectively the sets of maps defining the two given cylinders. From Proposition 1.6, $N$ is finitely generated, then up to enlarging $F_{N}$, we can assume that $F_{N}$ generates $N$ (in fact, in the proof of Proposition 1.6, it is proved that necessarily, $F_{N}$ generates $N$ ). Let $\mathcal{A}=\mathcal{A}_{H} \times \mathcal{A}_{N}$. Let us choose, for each $h \in H$, a pre-image $\tilde{h} \in \Gamma$ for the quotient map. Let $\widetilde{H}$ be the set of these elements in $\Gamma$. Let $F$ be the finite subset of $\Gamma$ defined by $F=\left\{\tilde{h} . n, h \in F_{H}, n \in F_{N}\right\}$. Let $M$ be the following set of maps:

$$
\begin{array}{r}
M=\left\{(m: F \rightarrow \mathcal{A}), \exists m_{H} \in M_{H}, \forall n \in F_{N}, m(\cdot . n)_{1}=m_{H} ;\right. \\
\left.\forall h \in F_{H}, m(\tilde{h} \cdot)_{2} \in M_{N}\right\},
\end{array}
$$

where the subscripts 1 and 2 denote the coordinates in the product $\mathcal{A}=\mathcal{A}_{H} \times \mathcal{A}_{N}$. Consider the cylinder defined by $F$ and $M$, and the associated subshift of finite type, $\Phi$. We need the following lemma.

Lemma 4.5. For any $\sigma \in \Phi$, there is at most one element $\gamma \in \Gamma$ such that $\sigma(\gamma)=$ $\left(\$_{H}, \$_{N}\right)$.

Proof. We first prove that for any $\sigma \in \Phi$, there is at most one left coset of $N, \tilde{h} N$, such that $\forall n \in N, \sigma(\tilde{h} . n)_{1}=\$_{H}$. By definition of $M$, if $n \in F_{N}, n_{0} \in N$, then $\sigma\left(\tilde{h} . n_{0} . n\right)_{1}$, the first coordinate of $\sigma\left(\tilde{h} . n_{0} . n\right)$ only depends on $\tilde{h}$ and $n_{0}$. But $F_{N}$ was chosen generating $N$, hence $\sigma\left(\tilde{h} . n_{0} . n\right)_{1}$ only depends on $\sigma(\tilde{h})$. By definition of $M$, the map $h \in H \mapsto \sigma(\tilde{h})_{1}$ is in $\Phi_{H}$, and therefore, by the special symbol property, there is at most one value of $\tilde{h}$ where it takes the value $\$_{H}$, this proves the first step of the lemma.Now, as the map from $N$ to $\mathcal{A}_{N}$ defined by $\left(n \mapsto \sigma(\tilde{h} . n)_{2}\right)$ is in $\Phi_{N}$, if $\tilde{h}$ is such that $\sigma(\tilde{h} . n)_{1}=\$_{H}$, there is at most one $n \in N$ such that $\sigma(\tilde{h} . n)_{2}=\$_{N}$. This proves the lemma.

Now, we define the map $\pi$ so that it sends a element $\sigma \in \Phi$ on the point at infinity, if $\sigma$ does not contain the symbol $\left(\$_{H}, \$_{N}\right)$, and on $\gamma \in \Gamma$ if $\sigma(\gamma)=\left(\$_{H}, \$_{N}\right)$. The map $\pi$ is well defined, and gives a finite presentation with special symbol of $\Gamma \cup\{\infty\}$.

Proof of Proposition 4.3. Assume that $\Gamma$ has the property of special symbol, and let $\mathcal{A}_{\Gamma}, \$_{\Gamma}, \mathcal{C}_{\Gamma}, \Phi_{\Gamma}$ the alphabet, special symbol, cylinder, and subshift of finite type associated. The cylinder is defined, as before, by two sets: $F_{\Gamma} \subset \Gamma$ and $M_{\Gamma} \subset \mathcal{A}^{F_{\Gamma}}$. We consider $\gamma_{1}, \ldots, \gamma_{n}$ a set of representatives of left coset of $G$ in $\Gamma$, and we choose $F=\left(\bigcup_{i=1}^{n} \gamma_{i}^{-1} F_{\Gamma}\right) \cap G$, a finite subset of $G$. We set $\mathcal{A}=\left(\mathcal{A}_{\Gamma}\right)^{n}$ and $M \subset \mathcal{A}^{F}$ is the set of the maps $m$ from $F$ to $\left(\mathcal{A}_{\Gamma}\right)^{n}$ such that there exists $m_{\Gamma} \in M_{\Gamma}$ whose translates $\gamma_{i}^{-1} m_{\Gamma}$ coincide with the $i$ th coordinate of $m$. Those three choices define 
a subshift of finite type $\Phi \subset \mathcal{A}^{G}$. By definition of $M$, one sees that there is a natural map $\Phi \rightarrow \Phi_{\Gamma}$ which consists of defining $\sigma_{\Gamma}(\gamma)$ by the $i$ th coordinate of $\sigma\left(\gamma_{i}^{-1} \gamma\right)$ if $\gamma$ is in the coset $\gamma_{i} G$. This map is a bijection, its inverse being the map that associates to $\varphi \in \Phi_{\Gamma}$ the element $\sigma \in \Phi$ whose $i$ th coordinate coincide with $\gamma_{i}^{-1} \varphi$. Therefore, one has maps $\Phi \rightarrow \Gamma \cup\{\infty\} \rightarrow G \cup\{\infty\}$, the second map being identity on $G$ and sending each $\gamma_{i}$ to 1 . At this point we do not have a special symbol, but, by property of $\Phi_{\Gamma}$, an element of $\Phi$ can take a value in $\mathcal{A}$ which has $\$_{\Gamma}$ among its coordinates, only once. Hence, by renaming each of those symbol by a single one $\$$, we get the expected presentation with special symbol.

Conversely, it suffices to see that the intersection of all the conjugates of $G$ is of finite index in $\Gamma$ (hence it has the special symbol property). It is normal and of finite index in $\Gamma$, and we can apply Proposition 4.2.

\section{References}

[1] B. H. Bowditch, Relatively hyperbolic groups. Preprint 1999. http://www.warwick.ac.uk/ masgak/papers/bhb-relhyp.pdf

[2] B. H. Bowditch, Geometrical finiteness with variable negative curvature. Duke Math. J. 77 (1995), 229-274. Zbl 0877.57018 MR 1317633

[3] M. Coornaert and A. Papadopoulos, Symbolic dynamics and hyperbolic groups. Lecture Notes in Math. 1539, Springer-Verlag, Berlin 1993. Zbl 0783.58017 MR 1222644

[4] M. Coornaert and A. Papadopoulos, Horofunctions and symbolic dynamics on Gromov hyperbolic groups. Glasg. Math. J. 43 (2001), 425-456. Zbl 1044.20027 MR 1878587

[5] F. Dahmani, Classifying spaces and boundaries for relatively hyperbolic groups. Proc. London Math. Soc. (3) 86 (2003), 666-684. Zbl 1031.20039 MR 1974394

[6] F. Dahmani, Combination of convergence groups. Geom. Topol. 7 (2003), 933-963. Zbl 1037.20042 MR 2026551

[7] F. Dahmani, Les groupes relativement hyperboliques et leurs bords. Ph.D. thesis, IRMA Strasbourg, Strasbourg 2003.

[8] F. Dahmani, Accidental parabolics and relatively hyperbolic groups. Israel J. Math. 153 (2006), 93-127. MR 2254639

[9] B. Farb, Relatively hyperbolic groups. Geom. Funct. Anal. 8 (1998), 810-840. Zbl 0985.20027 MR 1650094

[10] D. Fried, Finitely presented dynamical systems. Ergodic Theory Dynam. Systems 7 (1987), 489-507. Zbl 0652.54028 MR 922362

[11] M. Gromov, Hyperbolic groups. In Essays in group theory, Math. Sci. Res. Inst. Publ. 8, Springer, New York 1987, 75-263. Zbl 0634.20015 MR 0919829

[12] G. A. Hedlund, The dynamics of geodesic flows. Bull. Amer. Math. Soc. 45 (1939), 241-260. JFM 65.0793.02 
[13] P. Koebe, Riemannsche Mannigfaltigkeiten und nichteuklidische Raumformen. II: Allgemeines und niedere Raumformen. Sitzungsber. Preuss. Akad. Wiss. Berlin 1928 (1928), Phys.-math. Kl., XXIII, 345-384. JFM 54.0604.02

[14] M. Morse and G. A. Hedlund, Symbolic dynamics. Amer. J. Math. 60 (1938), 815-866. Zbl 0019.33502 MR 1507944

[15] M. Morse and G. A. Hedlund, Symbolic dynamics II. Sturmian trajectories. Amer. J. Math. 62 (1940), 1-42. Zbl 0022.34003 MR 0000745

[16] C. Series, Symbolic dynamics for geodesic flows. Acta Math. 146 (1981), 103-128. Zbl 0488.58016 MR 594628

[17] A. Yaman, A topological characterisation of relatively hyperbolic groups. J. Reine Angew. Math. 566 (2004), 41-89. Zbl 1043.20020 MR 2039323

[18] A. Yaman, Boundaries of relatively hyperbolic groups. Ph.D. thesis, University of Southampton, Southampton 2003.

http://www.math.uni-bonn.de/people/yaman/papers/Thesis.pdf

Received October 19, 2007; revised November 26, 2007

F. Dahmani, UPS, IMT (Institut de Mathématiques de Toulouse), 31062 Toulouse, France

E-mail: dahmani@math.ups-tlse.fr

A. Yaman, CRM Apartat 50, 08193, Bellaterra, Spain

E-mail: ayaman@crm.es 